\title{
¿Cómo gestionar, en tiempos de crisis, proyectos de transformación digital basados en innovaciones disruptivas para una organización?
}

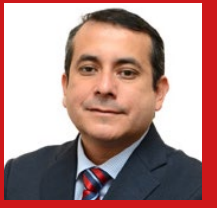

César Pino

PhD. en Management

Especialista en Estrategia de negocios

Universidad Austral de Chile

Email: cesarpino@uach.cl
La pandemia global producto

de COVID-19 y sus significativas

consecuencias sociales y económicas

ha generado un nuevo escenario de

incertidumbre para las empresas y

emprendedores. Esta situación puede ser abordada a través de la implementación de proyectos innovadores de

transformación digital que se sustentan en la reconfiguración estratégica de las capacidades dinámicas existentes en una organización, para lo cual se presentan recomendaciones específicas.

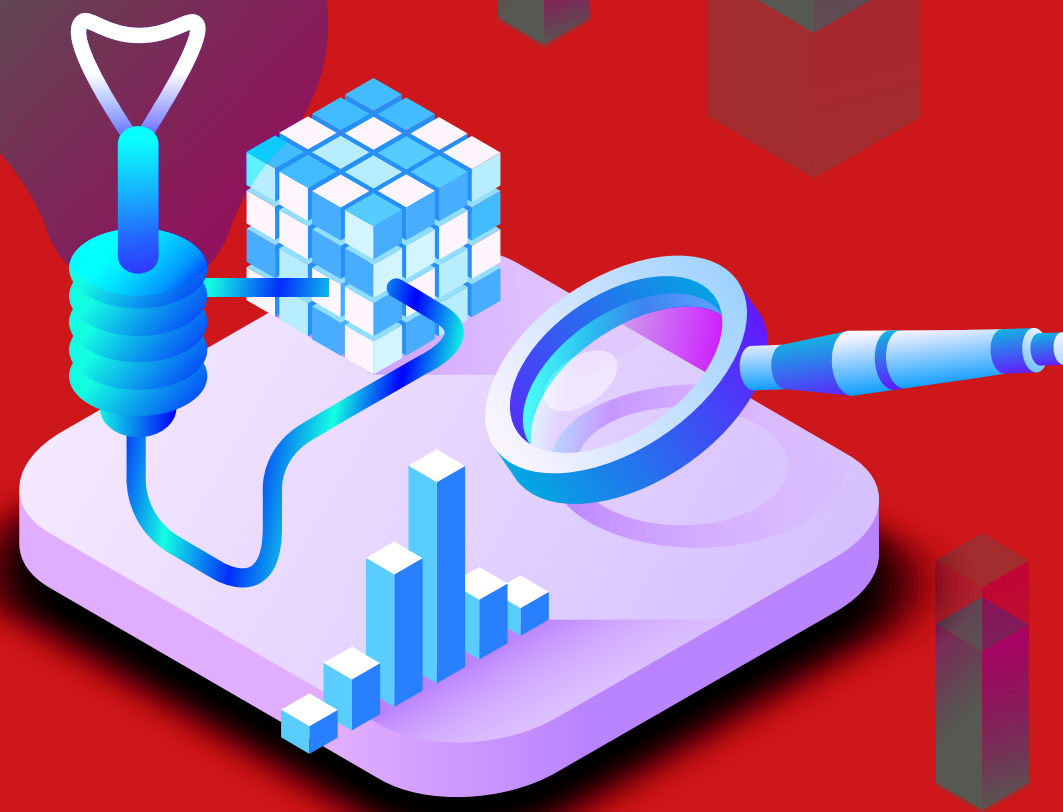

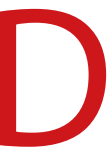
urante el 2020, la emergencia sanitaria mundial ha impuesto un escenario muy distinto al observado por las organizaciones en crisis económicas de décadas pasadas, como la "crisis subprime" del 2008. La intensa variabilidad de los mercados presiona a estados, empresas y emprendedores hacia un cambio en el rumbo estratégico que tenían planificado para los próximos años. Esto no es una tarea sencilla para el "top management" de una organización, ya que la decisión de continuar o desechar proyectos de innovación, se encuentra supeditada a los niveles de liquidez corporativos y limitadas posibilidades de apalancamiento financiero.

El desarrollo de proyectos de innovación puede hoy ser la clave para la subsistencia de la empresa, lo que permitiría, por una parte, optimizar el uso y control de los re- cursos y capacidades de la organización, y por otra, crear nuevas líneas de negocios para capturar nuevas oportunidades emanadas de los cambios en los mercados (Pino, Felzensztein, Zwerg-Villegas \& Arias-Bolzman, 2017)

Aun cuando, los beneficios de implementar proyectos de innovación o de transformación son ampliamente conocidos por la empresa, surge la interrogante respec- 
to a ¿cómo estos proyectos pueden ser gestionados en tiempos con profundos cambios?

Cambios como los observados en estos tiempos de crisis, solo son comparables a un escenario descrito cinematográficamente como "una tormenta perfecta" (en alusión a la película "The Perfect Storm" del año 2000 y protagonizada por George Clooney). En esta película una megatormenta es generada a partir de dos anomalías atmosféricas que colisionan y se funcionan entre sí, una tormenta de origen continental frío y una tormenta de origen oceánico cálido. Siguiendo esta analogía cinematográfica, las empresas latinoamericanas han tenido que enfrentar un escenario al que confluyen dos grandes fuerzas $y$, en este nuevo contexto, las firmas deben tomar la importante decisión respecto a desarrollar proyectos de innovación que les permitan asegurar su sostenibilidad futura.

Estas dos fuerzas son, por una parte, las limitadas capacidades y habilidades técnicas del capital humano en las organizaciones que, además, han debido adaptarse a nuevas condiciones para desarrollar sus labores a distancia, y, por otra, la aceleración de la adopción tecnológica de plataformas propias de la cuarta revolución industrial.

Un estudio realizado por Banco Interamericano de Desarrollo (2020) enfocado en las competencias y habilidades del capital humano de empresas latinoamericanas en el contexto de la cuarta revolución industrial, evidencia interesantes resultados sobre una muestra de 1.100 empresas en Argentina, Brasil, Colombia, Chile y Méxi$\mathrm{co}$, respecto del estatus de ambas fuerzas.

La primera fuerza corresponde a las capacidades y habilidades del capital humano en las organizaciones, el que además ha tenido que adaptarse al desarrollo del trabajo a distancia. Este estudio señala que, por una parte, un $24 \%$ de las empresas encuestadas había incorporado el trabajo a distancia hasta el año 2019, y un 33\% espera que esta tendencia se masifique en el futuro. Y, por otra, que 3 de cada 10 empresas señalan que existen faltantes de capacidades y habilidades en sus dotaciones actuales. Entre las capacidades que más escasean en las empresas están las habilidades STEM (ciencia, tecnología y matemáticas) con un $38 \%$ de faltante de esas capacidades, las habilidades de contenidos (comunicacionales y alfabetización digital) con un $36 \%$ y las habilidades de procesos (pensamiento crítico y automonitoreo) con un $35 \%$, estimándose que la demanda por estas capacidades se duplique en los próximos 5 años. Es importante señalar que estas habilidades faltantes son la base para desarrollar proyectos de transformación digital tendientes a implementar innovaciones disruptivas en las organizaciones.

La segunda fuerza corresponde a la aceleración de adopción tecnológica de plataformas propias de la cuarta revolución ternacional es extremadamente limitado (e incluso sesgado) respecto de tecnologías innovadoras en el mercado.

Desde el management, existe una perspectiva estratégica que constituye la base teórica para desarrollar proyectos de transformación en escenarios con cambios acelerados como los descritos. Esta perspectiva se fundamenta en el concepto de capacidades dinámicas (DC) que corresponde a la habilidad que posee la organización para integrar, construir y reconfigurar las competencias internas y externas de la misma, en función de ambientes que cambian rápidamente (Teece, Pisano \& Shuen, 1997; Eisenhardt \& Martin, 2000).

\section{"Aun cuando, los beneficios de implementar proyectos de innovación o de transformación son ampliamente conocidos por la empresa, surge la interrogante respecto a ¿cómo estos proyectos pueden ser gestionados en tiempos con profundos cambios?"}

industrial. Este estudio señala, por una parte, que las empresas de servicios (ES) tienen mayores porcentajes de adopción de tecnologías 4.0 por sobre las empresas productivas (EP), principalmente en torno a: computación en la nube $(78 \%$ en ES y $55 \%$ en EP), plataformas digitales y servicios móviles (76\% en ES y $61 \%$ en EP), y, ciberseguridad ( $63 \%$ en ES y $40 \%$ en EP). Sin embargo, se espera un mayor nivel de crecimiento de la adopción futura hacia una segunda oleada de tecnologías como son la inteligencia artificial y big data (análisis de datos), manteniéndose solo la ciberseguridad, la que sería mucho más masiva en el futuro. Lo relevante de este estudio es que muestra que la cuarta revolución industrial no se detiene en América Latina y que, por el contrario, avanzaría a pasos acelerados hacia nuevas tecnologías.

Emprender proyectos de transformación bajo un escenario en el que confluyen estas dos fuerzas, puede ser una tarea titánica de enfrentar para el equipo gerencial. Más aún, cuando no existen muchas experiencias empresariales que pudiesen orientar y cuando el conocimiento que existe en compañías que ofrecen servicios de consultoría independiente a nivel in-
Basado en la experiencia de liderar un proyecto de transformación digital en una universidad chilena (entre los años 2017-2020), me permito hacer recomendaciones que pudiesen orientar a quienes deben enfrentar este desafío de gestionar proyectos de innovación en tiempos de crisis como los que se viven actualmente. Estas recomendaciones se hacen en consideración a dos limitantes que aumentan la complejidad de este desafío. La primera tiene que ver con el hecho de que un proyecto de transformación digital a veces no es la continuidad de la tecnología existente sino que más bien corresponde a la implementación una innovación disruptiva, por lo que la organización no está preparada para enfrentar aquello en lo que no conoce, $y$, la segunda, tiene que ver con la inexistencia de experiencias de transformación en el sector educación superior chileno respecto de la adopción de tecnologías orientadas a la nube en organizaciones de similar naturaleza. Este proyecto nace para responder al requerimiento estratégico de modernizar la gestión manifestado por diferentes administraciones universitarias desde hace poco más de una década y su principal objetivo es implementar una innovadora 
plataforma tecnológica de gestión para la optimización integrada de los procesos de planificación, administración y control de recursos en el área de recursos humanos y área financiera.

La clave está en adoptar una correcta base teórica desde el campo disciplinar de la estrategia como la que ofrece DC para orientar la gestión de proyectos de innovación en torno a tres ejes: una correcta planificación del proyecto, el desarrollo de un análisis técnico agnóstico e independiente de sesgos hacia una tecnología específica y organizar idóneamente el proyecto para una controlada ejecución de este. A continuación, las recomendaciones específicas por cada eje:

\begin{tabular}{|c|c|}
\hline Eje & Recomendaciones específicas \\
\hline $\begin{array}{l}\text { Planificación } \\
\text { del proyecto. }\end{array}$ & $\begin{array}{l}\text { - Definir claramente de los objetivos y los alcances del proyecto, } \\
\text { ya que siempre existe la tentación de imputar al proyecto } \\
\text { requerimientos que nacen de áreas diferentes a la del foco } \\
\text { principal del proyecto. } \\
\text { - Creer con convicción en esos objetivos y no embarcarse en un } \\
\text { proyecto que no le convence. } \\
\text { - Dimensionar el estado del recurso humano de los potenciales } \\
\text { - Inverios del proyecto (resistencia al cambio). } \\
\text { gastos no contemplados en el futuro, sobre todo si esta } \\
\text { planificación se hace con apoyo externo. }\end{array}$ \\
\hline $\begin{array}{l}\text { Análisis } \\
\text { técnico } \\
\text { agnóstico. }\end{array}$ & $\begin{array}{l}\text { - No es recomendable dejar el } 100 \% \text { del análisis en manos del } \\
\text { - } \quad \text { Romper el "isomorfismo estratégico" en la forma de abordar } \\
\text { estos proyectos por parte de la empresa. Muchas organizaciones } \\
\text { desarrollan estos proyectos simplemente porque otros } \\
\text { (empresas competidoras) lo hicieron antes. } \\
\text { - Realizar un análisis agnóstico respecto de los beneficios y } \\
\text { limitantes que posee un producto tecnológico versus otro. }\end{array}$ \\
\hline $\begin{array}{l}\text { Organización } \\
\text { idónea para } \\
\text { una } \\
\text { adecuada } \\
\text { ejecución del } \\
\text { proyecto. }\end{array}$ & 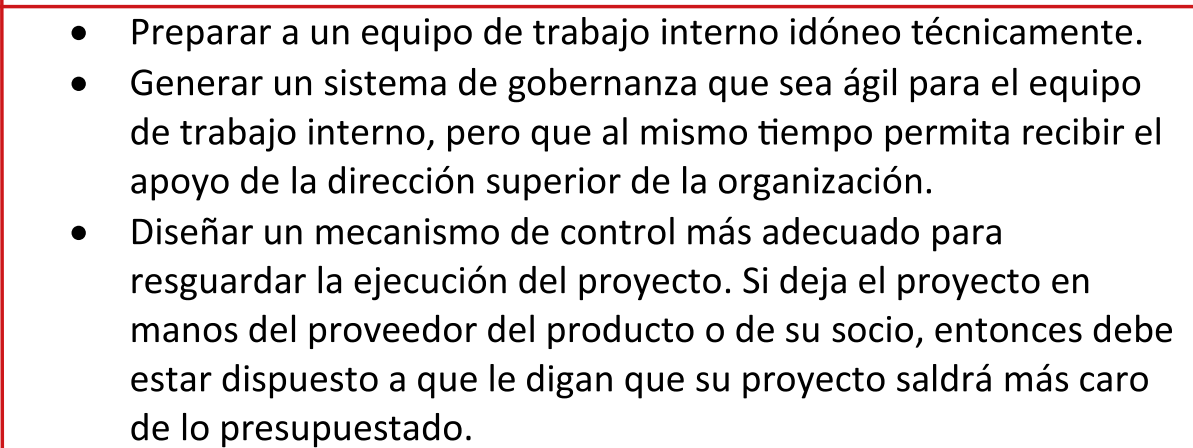 \\
\hline
\end{tabular}

Referencias

- Basco A., De Azevedo B., Harraca M. \& Kersner S. (2020), “Competencias y habilidades en la cuarta revolución industrial”, Banco Interamericano de Desarrollo.

• Eisenhardt K. y Martin J. (2000), “Dynamic Capabilities: What are They?”, Strategic Management Journal, Vol. 21, №10/11: pg. 1105-1121.

- Pino, C., Felzensztein, Ch., Zwerg-Villegas, A. M. y Arias-Bolzman, L. (2017). Non-technological innovation: Market performance of exporting firms in South America. Journal of Business Research. Vol. 69, Issue 10, 4385-4393.

•Teece D., Pisano G. y Shuen A. (1997), “Dynamic Capabilities and Strategic Management”, Strategic Management Journal, Vol. 18, N²7: pg. 509-533. 